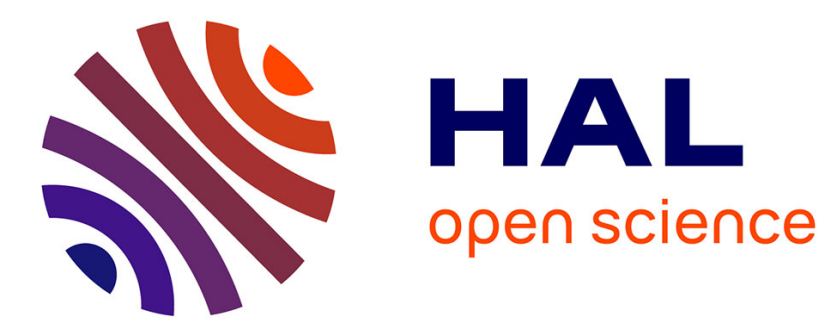

\title{
Pour une sociologie historique des sciences de gouvernement
}

\author{
Martine Kaluszynski, Olivier Ihl
}

\section{To cite this version:}

Martine Kaluszynski, Olivier Ihl. Pour une sociologie historique des sciences de gouvernement. Revue française d'administration publique, 2002, 102, pp.229-243. halshs-00343193

\section{HAL Id: halshs-00343193 \\ https://shs.hal.science/halshs-00343193}

Submitted on 1 Dec 2008

HAL is a multi-disciplinary open access archive for the deposit and dissemination of scientific research documents, whether they are published or not. The documents may come from teaching and research institutions in France or abroad, or from public or private research centers.
L'archive ouverte pluridisciplinaire HAL, est destinée au dépôt et à la diffusion de documents scientifiques de niveau recherche, publiés ou non, émanant des établissements d'enseignement et de recherche français ou étrangers, des laboratoires publics ou privés. 


\section{POUR UNE SOCIOLOGIE HISTORIQUE DES SCIENCES DE GOUVERNEMENT}

Olivier IHL

Professeur à l'IEP

de Grenoble (CERAT)

\author{
Martine KALUSZYNSKI
}

Chargée de recherche au CNRS

CERAT-IEP de Grenoble

Comment la conduite de l'action publique est-elle devenue objet de science? Répondre à cette question implique de prendre acte d'un préalable. Pour qu'il en soit ainsi, il a fallu que les affaires de l'État deviennent chose commune. D'un côté, qu'elles quittent l'antre de la Cour où jusqu'à la fin du XVII ème siècle, il était d'usage de parler d' «art de gouvernement »; un art assimilé, par les Miroirs des Princes (speculum regni) à des pratiques initiatiques bientôt couvertes du sceau de la raison d'Élat '. De l'autre, qu'elles «décrochent» de la pensée théologique, avec ses filiations el ses traditions attendues. Une désacralisation qui seule autorisait de nouveaux concepts à se substituer à la pesante glose sur les «mystères» de l'État. Avec l'avènement en Europe des monarchies absolutistes, avec le développement d’administrations monopolisant les fonctions gouvernementales, les choses changent. Ce n’est plus le secret qui légitime le pouvoir. C'est la science. Avec son exigence d'intelligibilité. Puis, son exigence de rationalité. Nombreux vont être les réformatcurs s'efforçant de constituer leurs savoirs en "sciences gouvernementales ». Des savants évidemment, mais aussi des administrateurs, des philanthropes, des mécènes, des hommes de lettres, des magistrats... Souvent sans sćpulture académique, ces figures ont eu un rôle déterminant. Elles ont imposé, sous couvert de projets réformistes, de nouvelles connaissances au coeur de l'action publique. Reconstituer leur rôle ne revient pas à réparer une injustice. Il s’agit plutôt d’analyser l'entrée en jeu de savoirs dont participent les dispositifs qui, encore de nos jours, encadrent le social. Plus exactement : de suivre leur métamorphose en ingénierie d’État mais aussi de comprendre leur institutionnalisation sous la forme de «disciplines » et parfois de «communautés açadémiques». Tel est finalement l'enjeu de l'attention nouvelle qu'historiens el politistes 2 portent aux concepts comme aux savoir-faire. aux

1. Sennelart (M.), Les arts de gowerner. Du régiment médiéval au concept de gouvernement. Paris, Seuil, (coll. "Des travaux "), 1995.

2. Notamment au CERAT-CNRS de l'IEP' de Grenoble. $\Lambda$ côté de cette équipe, if laut signaler le groupe ACI du CURAPP à l'Université de Picardie qui travaille sur « Les sciences camérales : activités pratiques et 
transactions comme aux concurrences qui forment la trame des pratiques de gouvernement. La question avait déjà retenu, dès 1911, l'attention de Max Weber dans ses premiers travaux sur la bureaucratie ${ }^{3}$. À ses yeux, deux dynamiques étaient particulièrement importantes: la professionnalisation des agents mis au service de l'État; la formalisation des savoirs à partir desquels l'administration revendique une supériorité d'action. C'est dire s'il importe ici de croiser au moins deux types de curiosité : l'une a trait au travail de rationalisation des conditions d'intervention publique, l'autre porte sur les usages qui sont venus justifier et produire de telles « spécialités ».

\section{DES SCIENCES CAMÉRALES AUX SCIENCES DE GOUVERNEMENT}

Le caméralisme, avec sa vision administrative du politique, en fournit une illustration. Rappelons le : en 1727, Friedrich Wilhem I ordonne la création d'une chaire Oeconomie, Polizey und Kammer-Sachen à l'Université de Halle. Peu après, une autre chaire est fondée à l'Université de Frankfurt. C'est le début du développement académique du caméralisme et de la Polizeiwissenschaft. Coïncidant avec la centralisation des «maisons princières» des Hohenzollern et des Habsbourg, ces disciplines visaient à renouveler la formation des futurs agents d'État. Plusieurs textes étaient apparus quelques décennies plus tôt pour revendiquer un tel changement dans les gouvernements nés sur les décombres du Saint Empire romain: de Ludwig von Seckendorff, avec son Teutscher Fürsten Staat (1656) à Wilhem von Schröder et son Fürstliche Schatz-und Rent Kammer (1686). Des entreprises dont la compréhension a toutefois beaucoup souffert des lectures académiques opérées aux $X X^{\text {ème }}$ et $X X^{\text {ème }}$ siècles, notamment par les économistes ou récemment par les tenants d'une «science administrative » dans leur quête d'un temps inaugural ${ }^{4}$. C'est ainsi, comme l'a montré Mack Walker, que les processus de régulation auxquels se sont atlachés nombre de ces practioners ne relevaient pas de formes juridiques mais d'une rationalité proprement

histoire des dispositifs publics ", sous la direction de Frédéric Audren et Pascale Laborier. Son projet consiste à développer une analyse comparative des modalités d'exercice de l'administration des États absolutistes curopéens (France, États allemands et italiens) aussi bien au travers d'archives que des manuels de formation administrative au «bon gouvernement». Autre groupe de travail avec lequel des échanges sont noués : celui présidé par Fabio Rugge au sein de l'Institut international des sciences administratives (IIAS), à Bruxelles, sur l'histoire de la science administrative, qui travaille en collaboration avec la revue Storia Amministrazione Costituzione (publiée par Il Mulino) et le Jahrbuch für europäische Verwaltungsgeschichte (publié par Nomos Verlag). De cet auteur, on lira récemment «Lc lcggi "Bassanini": continuità e innovazioni del riformismo amministrativo », Storia Amministrazione Costituzione, 4, 1997, p. 80-90 ; «Restoring Administration After a War. Some Reflections on Historical Experience », dans Thiedeck (F.), Vilella (G.) (dir.). Restaurer les capacités de l'administration et de la société civile après un conflit interne ou externe, Bruxelles. IISA, 1999, p. $65-74$.

3. Le chapitre «Bürokratie" figure, rappelons-le, dans la $3^{\text {èmc }}$ partie intitulée « Les types de domination » de son célèbre ouvrage Wirtschaft und Gesellschaft, Grundriss der Sozialokonomik (Tubingen : Mohr, 1955), p. 650-678.

4. Sur cette instrumentalisation du savoir caméral au XIXène siècle, cf. Friedrich (Carl J.), « The Continental Tradition of Training Administrators in Law and Jurisprudence », The Journal of Modern History, 11, 2, juin 1939, p. 129-148. Le Traité de science administrative, publié en 1966, par Mouton et l'École pratique des hautes études en offre un témoignage : au nom de la recherche d'ancêtres fondateurs, il rattache ces figures à une « pensée administrative » caractérisée par les postulats essenticls de l'histoire des idées (unité et cohérence des ceuvres, force propre de l'originalité créatrice, primat accordé au scns déclaré). Une lecture effectuće au détriment d'une étude véritable des règles de formation de ces objets, concepts ct théories. Au 
bureaucratique ${ }^{5}$. Contrairement au discours des philosophes traitant des doctrines de la «bonne gestion » sur la base d'une exégèse des "grands auteurs », c'est avant tout un contenu pratique qui fut donné à ces prétendues «sciences" : celui d'améliorer les techniques et objets de l'action gouvernementale. En somme, ce qui s'imposait là, c'est une autre conception de l'exercice de la puissance. La conduite des hommes et des choses? Elle s'exerce non par l'observance du droit, fut-il naturel, ou dans l'ombre portée de la philosophie, mais guidée par « l'expérience ». C'est-à-dire en se fondant sur des outils élevés au rang de garants, sinon de critères de l'action publique ${ }^{6}$. Un travail continu qui peut être assimilé à une fabrique gouvernementale de l'État.

De tels militantismes scientifiques n'aident pas seulement à penser l'histoire de l'administration (le fonctionnement par découpages sectoriels ou les niveaux d'action gouvernementale - les découpages territoriaux - qui sont encore les nôtres). Ils importent également pour comprendre la spécialisation de ces instruments de pensée dans leurs liens avec les formes de structuration de l'espace social. Un seul exemple : les concepts de "hiérarchie des offices" ou de «division du travail routinière » chez Seckendorff dans son Fürsten Staat. Ils tendent aussi bien à une plus grande efficience des formes d'organisation qu'à systématiser le principe de loyauté des agents administratifs. D'où son idée de les enfermer dans un nouvel « esprit de corps». Elle répond à la nécessité de construire l'autorité de la bureaucratie en la calquant sur le modèle aristotélicien du pater familias ${ }^{7}$. À l'évidence, aussi longtemps que l'on considèrera les écrits des «caméralistes » à leur seule valeur académique, soit en essayant d'y retrouver l'analogue des travaux contemporains sur l'« économie du développement » ou de «la planification » soit en s'efforçant d'y lire « une variante allemande du mercantilisme », il ne sera guère possible de comprendre les mobiles qui animaient ces figures.

Ne l'oublions pas : les premiers caméralistes vivaient au moment où le Saint Empire Romain germanique cessa d'être une entité constituée, démembrée qu'il était par des puissances nouvelles. Or, ce contexte de guerres religieuses et de reconstruction de l'État

détriment aussi des régularités et usages qui ont pu commander l'apparition de telles revendications de scientificité.

5 «Rights and Functions: The Social Categories of Eighteenth-Century German Jurists and Cameralists ", Journal of Modern History, 50, juin 1978, p. 234-251. L'auteur y étudie la différenciation d'une classe de "professionnels administratifs": il montre en particulier combien c'est l'autonomie croissante de leurs activités qui a «fait sortir ces hommes hors de la société à la poursuite d'une tâche professionnelle : celle de la guider et de la régler» (p. 234). Ainsi en va-t-il du «management » de la justice (Handhabung) : il s'est séparé de la simple jurisprudence (Rechtsgelehrsamkeit) à partir d'un tcl schéma. De Macker, on lira également Johann Jakob Moser and the Holy Roman Empire of the German Nation, Chapel Hill, University of North Carolina Press, 1981.

6. Sur l'importance des problèmes de conduite des organisations ou de rationalisation de la décision chez ces administrateurs qui se targuaient de scientificité, voir le texte injustement oublié de Hubcrt C. Johnson "The Concept of Bureaucracy in Cameralism», Political Science Quarterly, 79, 3, septembre 1964, p. 378-402.

7. Sur la transformation de cette étiquette curiale en un protocole d'État, creuset d'un gouvernement par les récompenses honorifiques et les signes de mérite, voir Olivier Ihl, «Les rangs du pouvoir. Régimes de préséances et bureaucratie d'État dans la France des XIX ${ }^{\text {eme }}$ et XX ${ }^{\text {ènc }}$ siècles », dans Deloye (Y.), Haroche (C.) et Ihl (O.) (dir.), Le protocole ou la mise en forme de l'ordre politique, Paris, L'Harmattan, 1996, p. 233-263. Cet ouvrage est issu d'un colloque organisé en juin 1995 par l'AFSP à l'Institut International d'Administration Publique, cela dans le cadre du Groupe de Recherche sur l'Etiquette et le Protocole Politique du département de Science politique de l'Université Paris I (Panthéon-Sorbonne). Et pour l'étude des usages politiques de la décoration dans l'univers des colonies, du même auteur, "Sous le regard de l'indigène. Le voyage du président Loubet en Algérie », dans Jean-William Dereymez, Olivier Ihl, Gérard Sabatier (dir.), Un cérémonial politique : les voyages des chefs d'État, Paris, L'Harmattan, 1998, notamment p. 226-233. Le texte est le fruit d'un colloque organisé à l'IEP par le CERAT le 9 novembre 1995 
posail des contraintes inédites, en particulier à ceux qui tentaient de renforcer la place que le gouvernement pouvait y tenir. Dans un monde où la pensée demeurait dominée par les référents juridiques et philosophiques, Melchior von Osse et ses successeurs concevaient, cux, le regimen dominativum comme devant organiser la société. Leurs savoirs? Ils l'élaboraient au service de princes certes toujours chrétiens mais dont la légitimité, face à la puissance des villes franches, de la noblesse ou de l'Église, reposait principalement sur une capacité administrative et militaire. D'où l'attrait de la « science de la police» dans un tel contexte. Elle promettait de dissocier le commandement administratif des règles de lignage et de fidélité chères à l'aristocratie foncière. La complexité des pays germaniques avant 1806, ni monarchie centralisée, ni confédération d'États indépendants, est donc essentielle dans l'émergence de celte «discipline». Émancipée du droit romain comme de la philosophie politique, celle-ci repose sur deux propositions embôtées: la première, dérivée du monde féodal, établit que le corps politique est un organisme; la seconde, née dans les milieux du négoce, pose que la société est fondée sur une division du travail. Un double référent - stabilité organique et spécialisation fonctionnelle - constamment mobilisé. Et déjà par les zélateurs des structures centrifuges (comme la Bavière ou la Saxe) désirant s'abstraire de la tutelle impériale. Absorbant certains concepts mais aussi certaines revendications du mouvement des Lumières, cette «science de la police » se transformera au XVIII ${ }^{\text {ème }}$ siècle en un académisme. En un savoir d'État destiné à promouvoir les seuls changements qui bénéficiaient au pouvoir des Princes.

Le caméralisme demeure donc hybride dans ses composantes: il amalgame des principes constitutionnels (Verfassungslehre), des conseils tactiques aux princes (Staatsklugheitlehre) et des idées plus génériques sur le gouvernement et l'administration (Staats und Verwaltungslehre) : comme l'idée d'un salariat permanent de professionnels issus des classes moyennes, comme le principe d'agents recrutés par examen plutôt que par patronage ou vénalité des charges ${ }^{8}$. Les disciplines universitaires devaient désormais tenir et contenir de telles «sciences» dans le cercle de leurs abstractions. Car cette académisation du savoir sur et dans l'État s'énonçait comme la découverte d'une sorte de providence laïque. L'amélioration du sort des «classes les plus nombreuses et les moins heureuses » n'était plus vue comme dépendant d'un certain arrangement de "l'économie » mais de l'action propre de l'État. D'une rationalité instrumentale que désignait alors une expression: celle d'un «ménagement public». Une formule par laquelle se trouvait consacrée l'ascendance des détenteurs d'un savoir technique ${ }^{9}$. Des serviteurs d'autant plus dociles qu' ennoblis par la tâche bureaucratique, ils devraient tout à ce magistère porteur d'une définition nouvelle du pouvoir.

8. Small (Albion), « Some contributions to the History of Sociology : approaches to objective economic and political science in Germany : Cameralism», American Joumal of Sociology, 29, 2, 1923, p. 158-165. Le terme de «police » doit être évidemment compris dans l'acception aristotélicienne de la promotion d'un «bon ordre » de la société. Sur l'emploi du terme, voir la clarification apportée par Paolo Napoli, «Police: la conceptualisation d'un modèle juridico-politique sous l'ancien régime ", Droits, 20, 1994, p. $183-196 \mathrm{cl} 21$, 1995 , p. $151-160$.

9. Pour une lecture qui donne la primauté au rélérent politique dans l'ćlude du caméralisme et, ce faisant, qui a largement renouvelé l'approche de ces milieux réformateurs, voir Tribe (K.), « Cameralism and the Science of Government », Journal of Modern Historn: 56, juin 1984, p. 263-284. 


\section{LA PRODUCTION DES SAVOIRS}

S'intéresser à ces figures de penseurs et de bureaucrates, mais aussi à ces revues, réseaux, théories ou disciplines aujourd'hui dédaignés, ce n'est pas céder à une passion érudite. C'est œuvrer à une histoire sociale, celle des formulations doctrinales et des revendications de scientificité dont s'est continûment enorgueillie la conduite du pouvoir bureaucratique. Une façon de se donner les moyens d'interroger les instruments de connaissance et de légitimation par lesquels se font puis se défont les modèles d'action gouvernementale, à l'échelle de l'Etat comme à celui des collectivités dites locales ${ }^{10}$. Car ces sciences de gouvernement, qu'elles soient oubliées ou toujours actuelles, décriées ou pourvues de titres académiques, ont apporté l'éclat de l'objectivité aux pratiques du gouvernement de la Cité ${ }^{11}$. Elles ont déjà au XIX ${ }^{\text {c̀mc }}$ siècle fait les délices des littérateurs. Que l'on songe au bureau des circonlocutions dans le roman The Little Dorrit de Charles Dickens. Censé détenir « la science entière du gouvernement ", ce département, le plus en vue dans la hiérarchie des pouvoirs, devait donner son consentement à toutes les affaires publiques, des plus importantes aux plus insignifiantes. De sorte que « its finger was in the largest public pie, and in the smallest public tart $» 12$.

École, fiscalité, presse, locomotion, monuments historiques, état civil, hygiène, élections, domanialité, célébrations, propagande: les domaines d'intervention de ce « ménagement public » n'ont depuis cessé de s'étendre. Et avec eux les connaissances, à la fois pratiques et anonymes, fonctionnelles et édifiantes, qui leur servent de moyens d'action. Celles-ci se déploient à travers tout un ensemble d'instruments de classement, de mesure et d'enquête (archives, organigrammes, recensement, cartographie, formulaires, règlements...). Entreprendre de les étudier, c'est poser la question des supports de diffusion qui les accréditent (revues, congrès, chaires professorales, etc.) ; c'est revenir sur les procédures académiques qui les certifient, interroger les mobilisations de soutien et de défiance qu'elles ont suscitées. C'est, plus encore, évaluer l'efficacité qui leur est propre, celle qu'elles réclament au nom des catégories d'intervention publique, celle aussi qu'elles consacrent en tant que techniques particulières de légitimation. Ainsi, le « docteur » Jules Fournet, auteur d'un article sur l'« Influence de la science en général et de la science médicale en particulier sur la raison publique et le gouvernement des sociétés » en appelle à une science d'État fondée sur les lumières de la médecine : une expertise qui «peu à peu ramènerait les hommes, mieux que des préceptes et des

10. Sur l'action propre des structures bureaucratiques «en local », voir Dumons (B.), Pollet (G.) (dir.), Élites et pouvoirs locaux. La France du sud-est sous la Troisième République, Presses Universitaires de I yon, 1999, et le numéro spécial « Le temps des mairies », Politix, vol. 14, n 53, 2001.

11. C'est de ce dessein qu'est né le colloque qui s'est tenu à Grenoble, les $15-17$ novembre 2000 sur « La formation des sciences de gouvernement en Europe XIX ${ }^{\text {ème }}-X^{\text {éme }}$ siècles " au conseil général de l'Isère el à l'IEP de Grenoble. Il réunissait une vingtaine de chercheurs venus d'Allemagne, d'Italie et de France. Une publication des actes de cette rencontre est en cours sous la forme d'un numéro spécial de la Revue française de science politique (coordonnateurs : Rachel Vanneuville el Renaud Payre) et d’un ouvrage chez Économica, Les sciences de gouvernement. Ihl (O.), Kaluszynski (M.), Pollet (G.) (dir.). Certains développements reprennent ici des points évoqués dans l'introduction de l'ouvrage.

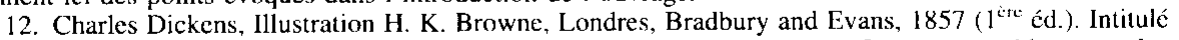
«Containing the Whole Science of Government», le chapitre 10 de ce roman-fleuve rassemble toutes les critiques que pouvait susciter le monde des commis-fonctionnaires de l'Angleterre viclorienne. Issu d'une famille modeste, Dickens fut, avant de se lancer dans la carrière des lettres, clerc de notaire puis, fin 1828 sténographe aux Doctors'Camons et reporter-sténographe au Parlement. Une expérience qui alimente largement sa critique du fonctionnement bureaucratisé de la monarchie des Hanovre-Windsor. 
rigueurs, à aimer l'autorité, à respecter ses sages lenteurs et à se défaire dans l'intérêt même du progrès, qu'ils ont raison de poursuivre, de ces impatiences désordonnées, de l'avenir qui détournent des longues préparations du présent, de ces oppositions de forces qui paralysent l'effort social et de ces alternances de morne inertie et de fougue révolutionnaire où les grands ressorts de l'État se brisent après s'être rouillés " ${ }^{13}$. On le constate : le terme de science est moins ici un constat qu'un problème. Moins un point d'arrivée qu'un point de départ: le creuset d'une interrogation sur les régimes d'objectivité associés au déploiement des universalismes de la puissance.

Régime d'objectivité : le terme pourra sembler abstrait. Pourquoi ne pas recourir plutôt à la notion de paradigme ? Dans son ouvrage publiée en 1962 (Structure des révolutions scientifiques, traduction française, Flammarion, 1983), Kuhn assimile - rappelons-le - la notion à un ensemble structuré de concepts, de théories et de techniques instrumentales. Un ensemble qui, dans une conjoncture donnée, indique les contours du monde que les savants peuvent interroger. Cependant, le terme demeure enfermé dans une vision psychologique. Il sous-estime l'influence des facteurs sociaux et des compétitions intellectuelles sur le développement de la recherche. La succession de ces paradigmes? Elle est présentée comme découlant de l'apparition de « faits nouveaux » qui restent inexplicables par l'ancien modèle théorique. Ce qui entraînerait, après une période d'incertitudes, un groupe d'individus « à l'imagination particulièrement fertile » à construire un nouveau cadre d'interprétation. Reste qu'une telle approche ne dit rien sur l'autorité sociale de ces outils : notamment sur la légitimité de ceux qui les commandent, les reçoivent ou s'en prévalent. Plus important : elle oublie combien les disciplines qui s'imposent font disparaître les causes qui les ont produites, devenant du même coup incompréhensibles par leur réussite même. Enfin, elle néglige le fait que ces dispositifs de connaissance dérivent pour une part du travail de praticiens : conseillers, avocats, bureaucrates, élus... Des ingénieries qui se forment au cœur de mouvements internationaux, à rebours donc des traits nationaux que la littérature scientifique aime à conférer aux "grandes inventions ${ }^{14}$.

Comment continuer à en douter ? Il importe d'élargir la question des processus de formation des savoirs d'État. Longtemps, l'histoire des sciences a privilégié une évolution gradualiste, avec l'idée d'un progrès s'effectuant de façon lente et régulière. Puis, l'approche en termes de paradigmes a pris le relais : le changement procéderait désormais de transformations brusques, celle des «mutations » de la recherche, celle aussi des pressions sociales qui favorisent le développement de nouvelles communautés savantes. L'analyse des "sciences » de gouvernement s'adosse plutôt à un schéma en mosaïque : avec une évolution à la fois distincte de toute continuité linéaire et dispersée entre des milieux professionnels et militants définis comme en rivalité pour imposer des domaines d'objets spécifiques comme des formes de savoirs conformes à leurs attentes. En somme, c'est une double logique qui prévaut: de spécialisation périphérique et de relèves concurrentielles.

Exemple de ces luttes entre producteurs de connaissances : l'appel à l'Académie des sciences par le gouvernement provisoire de 1848 lors des préparatifs des premières

13. Paris, V. Masson et fils, 1864,27 p. Extrait de la Revue britannique dans son numéro de décembre 1864.

14. Sur ces transferts contrôlés de savoir-experts concernant le gouvernement municipal, voir l'article de Renaud Payre, "The Science That Never Was: "Communal Science" in France, 1913-1949", Contemporary European History, II, 4, 2002, p. 529-547. 
élections au «suffrage universel ». Il peut s'analyser comme une revanche : celle des ingénieurs polytechniciens contre les tenants d'une économie politique hostile aux modèles mathématiques et arc-boutée au sein de l'Académie des sciences morales et politiques rétablie par le ministère Guizot ${ }^{15}$. Qu'on ne s'y méprenne pas : il ne s'agit nullement en avril 1848 de fonder une nouvelle science, celle des élections, simplement de forger — dans la précipitation — des techniques auxiliaires du pouvoir. D'abréger les opérations de collecte, de totalisation et de classement des voix recueillies pour faire face à un défi : accueillir et décompter neuf millions de voix ${ }^{16}$. Le rétablissement de l'Académie des sciences morales et politiques en 1832 s'était fait au nom d'une revendication de scientificité aux antipodes du modèle défendu par les membres de l'Académie des sciences : en cherchant ses bases théoriques dans l'économie politique mais aussi dans la philosophie de l'histoire. Contre cette définition de la science, antérieure au savant et dont ce dernier vient « dévoiler » les plans, une science chargée de dresser une diguc aux «passions » et aux « utopies », certains défendent, au contraire, les notions d'essai et d'expérimentation ${ }^{17}$. Leur vocabulaire? Ils l'empruntent aux manuels de physique ou de mécanique. Leurs idéaux? Aux publications comme le Discours sur le positivisme d'Auguste Comte, celles-là mêmes qui poussent Deslaurier, dans $L^{\prime}$ Éducation sentimentale, à un retentissant « il serait temps de traiter la politique scientifiquement!». À ceux-là, les défenseurs des «sciences morales et politiques » opposent une vigoureuse mise en garde : «Dans l'état présent de la science, et à la prendre telle qu'elle est communément enseignée, le gouvernement n'est pas à proprement parler une chose qui lui appartienne ${ }^{18}$. On ne saurait mieux dire. Si les connaissances sur la conduite « des hommes et des territoires » furent très tôt déduites d'un principe d'utilité (conduire les hommes de « la manière la plus rationnelle et la plus avantageuse possible ${ }^{19}$ ), celles-ci passaient par une appropriation académique de la notion de gouvernement. Une notion qui ne se ramène pas, elle-même, à l'administration en tant que telle, encore moins aux caractéristiques de la communauté politique, mais demeure appréhendée comme « un corps intermédiaire entre la loi fondamentale de l'État et la nation ». Un corps doté « d'une existence, d'une vie réelle qui anime tout l'État, pour que tous les membres puissent agir de concert et répondre à la fin pour laquelle il est institué $» 20$.

C'est pourquoi la thématique des sciences de gouvernement est construite à travers une approche spécifique : la sociologie historique du politique. Sans ouvrir ici de longs

15. Sur le rôle militant de ces academics (Charles Dunoyer, Charles Dupin, Joseph Garnicr ou Michel Chevalier), voir Martin S. Staum, «French Lecturers in Political Economy, 1815-1848: Varieties of Liberalism », History of Political Economy, 30.1(1998), p. 95-120.

16. Sur l'emprise des ingénieurs réformateurs sur l'organisation du suffrage universel, voir Olivier Ihl, “Une ingénierie politique. Augustin Cauchy et les élections du 23 avril 1848 », Genèses, 49, décembre 2002, p. $5-25$.

17. Sur cette figure sociale, voir Grattan-Guiness, «The Ingénieur-savant, 1800-1830: a Neglected Figure in the History of French Mathematics and Science », Science in Context, 6, 1995, p. 405-433.

18. Art. «Gouvernement» signé par l’économiste "guizotien» Charles Dunoyer, dans Charles Coquelin et Gilbert Guillaumin (dir.), Dictionnaire de l'économie politique, Paris, Guillaumin, 1853, T. 1, p. 835 .

19. Art. "Gouvernement», Dictionnaire des sciences politiques et sociales, Auguste Ott, Paris, J.P. Migne, 1854 , p. 150 . Une conception qui pousse à rationaliser les interactions que le gouvernement doit avoir « avec le corps de l'État, selon les rapports accidentels et particuliers par lesquels ce même État est modifié ».

20. Jean-Baptiste Robinet, Dictionnaire universel des sciences morale, économique, politique et diplomatique, Londres, Libraires associés, 1777, T. 20, p. 470. 
débats épistémologiques ${ }^{21}$, signalons que cette démarche vise à questionner la genèse du politique, entendue comme instance de régulation sociale. Elle vise à réhabiliter la longue durée des forma mentis qui sont au cœur de l'action publique. À saisir des moments propices à la redéfinition de ces hiérarchies et divisions du travail propres aux dispositifs d'encadrement bureaucratique, des moments dont l'analyse exige de combiner récits diachroniques et études comparatives ${ }^{22}$. Partant des problèmes du présent, elle cherche à comprendre les conditions de formation des institutions contemporaines, notamment les défis auxquels ces dernières sont confrontées, à partir d'une réflexion sur leurs circonstances d'élaboration. C'est ainsi un nouveau rapport de connaissance à l'objet qui est proposé. Son objectif ? Mettre en lumière les mécanismes qui sont au cœur de l'institutionnalisation de l'action publique ${ }^{23}$. La notion de processus est particulièrement féconde dans une telle perspective. Avec elle, il est possible d'historiciser les raisonnements à prétention scientifique. De faire des réalités administratives (concepts, institutions, règles) non seulement des instruments d'analyse mais des objets de réflexion. De s'interroger du coup sur leur valeur stratégique, sur les conflits qui ont accompagné leur avènement et, plus généralement, de retrouver les paramètres de leur existence aujourd'hui largement impensée.

Manifester une telle préoccupation ne consiste pas à reconduire le souci d'un commencement fondateur ou à traquer des relations cachées. Le propos est ailleurs. Il s'agit de rendre explicite ce qui demcure implicite et déjà à force d'être sous les yeux de tous. La familiarité est le premier obstacle à la compréhension des objets de pouvoir. L'approche socio-historique permet, elle, d'élargir nos cadres de référence. Elle favorise le comparatisme mais cette fois dans une double dimension : spatiale et temporelle. Si elle est historique, cette démarche n'entend pas lier pour autant passé et présent de façon mécanique. C'est pourquoi il faut surtout lire dans cette démarche une invitation : celle de contextualiser les catégories d'analyse qu'appelle l'examen de l'action publique au quotidien 24 .

L'histoire ne se répétant pas, cette curiosité se confie à un « raisonnement sociologique »: entre vérification historique et raisonnement expérimental ${ }^{25}$. Pour le dire autrement : au cœur de la pratique de gouvernement, la problématique de l'État ne se pose pas indépendamment, ou à côté, des jeux d'acteurs. Elle se présente, au contraire, à travers la succession ordonnée de leurs interactions. L'État n'y est pas abordé en termes de structure, mais à travers une théorie de l'action fondée sur l'activité propre des groupes et des représentations. Il y est défini, pour reprendre la terminologie wébérienne, comme une catégorie représentant des «espèces déterminées de la coopération hu-

21. Pour un approfondissement de ces questions, Deloye (Y.), Sociologie historique du politique, Paris, La Découverte (Coll. « Repères »), 1997

22. Sur cette méthodologie, voir Meyer (D.) et Skocpol (T.) (dir), States, Social Knowledge and the Origins of Modern Social Policies, Princeton (N.-J.), University Press of Princeton, 1996.

23. Voir, dans ce sens, la synthèse des travaux issus du colloque organisé à Grenoble en mai 1996 par l'association Ship (Sociohistoire du politique), Martine Kaluszynski, Sophic Wahnich (dir.), L'État contre la politique? Les expressions historiques de l'étatisation, Paris, L'Harmattan (coll. "Logiques politiques"), 1998.

24. Sur ces attendus, voir Pollet (Gilles), «Regards croisés sur la construction de la loi : d'une histoire sociale à une socio-histoire de l'action publique», in: Commaille (Jacques), Dumoulin (Laurence), Robert (Cécile), La juridicisation du politique. Leçons scientifiques, Paris, LGDJ, 1999.

25. Pour une présentation de ces controverses méthodologiques, voir Offerlé (M.). «L'Histoire des politistes », dans Favre (P.) et Legavre (J.-B.) (dir.), Enseigner la science politique, Paris, L'Harmattan, 1997. 
maine ${ }^{26}$. En somme, comme un type particulier de relation sociale. Une manière, on le voit, de rompre avec une vision essentialiste de l'État, de la nation et de ses institutions au profit de leurs manifestations socio-historiques, à la fois mouvantes et disputées. Loin d'être des entités englobantes et figées, ces dernières sont "prises » dans les actions de ceux qui les constituent. Elles sont sujettes à de multiples traductions et redéfinitions sclon les situations expérimentées. Signe que l'histoire est moins investie ici comme une méthode que comme une démarche : pour excentrer les interrogations, produire des liens entre les différentes manières d'aborder l'objet politique et ainsi mieux assumer l'interdisciplinarité que requiert l'étude de la gouvernementalité publique (v. annexe).

\section{LES DISCIPLINES DU POLITIQUE}

On l'aura compris : il s'agit moins ici de restitucr l'histoire d'une discipline en partant des représentations que ses défenseurs en ont ou de ses problématiques contemporaines que d'aller explorer des moments et des lieux : ceux au cour desquels ont pu s'organiser les entreprises de rationalisation qui ont pris le gouvernement des corps et des esprits pour motif. Les « arts » de gouvernement ont prétendu se hisser à la hauteur d'une « science». Au terme de quelles difficultés et de quelles tractations ? Sous le poids de quelles nécessités, à partir de quels contextes économique, social ou démographique? L'institutionnalisation de ces savoirs a supposé la mobilisation de soutiens spécifiques, elle a requis la mise en œuvre de stratégies et de supports de publicisation. Quels sont-ils ? Comment se constituent-ils ? Elle a également nécessité l'obtention de signes de reconnaissance et de ressources diverses pour transformer des préoccupations militantes en discours universitaire mais aussi en prétention académique ou en formulation doctrinale. De quelles «vertus » académiques jouissent les savoirs érigés en «sciences » du pouvoir? À quelles contestations se heurtent-elles lorsqu'elles finissent par se dévaluer?

Qu'il s'agisse de l'identification des personnes et des groupes, de la structuration de l'administration elle-même ou encore de la mise en forme de ses rapports avec la société civile, chaque nouvelle catégorie d'action publique fait surgir un ensemble de concepts et de lieux de formation. Des instruments de mesure et d'enquête, des parutions savantes, des chaires universitaires, des controverses doctrinales : à chaque fois, avec l'objectif de conférer au domaine d'intervention de l'État une légitimité spécifique. Finances publiques, préséances civiles et militaires, droit parlementaire, techniques commerciales, statistique de population, législation comparée : voilà les «sciences» de l'action gouvernementale que les XIX ${ }^{\text {eme }}$ et $X X^{\text {èmc }}$ siècles vont approfondir et spécialiser en les mettant au service de la bureaucratie ${ }^{27}$. Partant, ce sont de véritables disciplines universitaires qui seront élaborées. Plus autonomes, plus professionnalisées, ce sont d'elles que viennent en droite ligne les «spécialités » et autres «expertises » qui aujourd'hui encore trament l'administration du politique. Qu'il suffise d'évoquer le

26. Sur cette sociologic compréhensive de l'historicité de l'État, voir Deloye (Y.) et Voutat (B.) (đir.), Faire de la science politique, Paris, Belin (coll. «Socio-histoires »), 2002.

27. Racff (M.), The Well-ordered Police State: Social and Institutionnal Change through Law in the Germanies and Russia 1600-1800, New Haven (Conn.), Yale University Press, 1983. 
développement récent du «management public » promu nouveau Graal de la raison bureaucratique. N'est-ce pas en son nom que des réformes d'inspiration libérale prétendent aujourd'hui « réinventer le gouvernement » 28 ? Partisans d'une «perestroika américaine », Osborne et Gaebler représentent bien ces réformateurs soucieux de mobiliser, au nom de la dénonciation de la "pression fiscale " hommes politiques et administrateurs pour travailler à élargir les « régulations de marché». Cette nouvelle « forme entrepreneuriale » du gouvernement (new « entrepreneurial» form of government) se présente comme une compilation des expériences les plus «innovantes ». Elle traduit la puissance des activistes de cette «nouvelle science managériale » qui, depuis l'ère Reagan, se sont multipliés à travers tout le pays. S'appuyant sur la littérature spécialisée, notamment les travaux de James Q. Wilson sur la Bureaucracy, ils proposent un nouveau modèle d'action publique, à la manière finalement dont les practitioners de l'Angleterre des Tudor s'étaient élancés à l'assaut de « l'arithmétique politique » grâce à leurs travaux sur les effets d'escompte, les pompes à air ou les tables de mortalité 29 . C'est justement à comprendre les conditions de formation, de circulation et de développement de ces «sciences de gouvernement» dans l'Europe des XIX ${ }^{\text {ème }}$ et $\mathrm{XX}^{\text {ème }}$ siècles que s'attachent les chercheurs réunis par ce chantier de recherche.

\section{UNE SCIENCE DANS TOUS SES ÉTATS}

L'expression de «sciences de gouvernement » est restée longtemps métaphorique. Helvétius, par exemple, l'utilise dans un sens très restrictif : "S'il est très dangereux de toucher trop souvent à la machine du gouvernement (...), il est des temps où la machine s'arrête, si l'on n'y remet de nouveaux ressorts"; mais cela suppose une "sage hardiesse", c'est-à-dire "l'étude profonde de la science du gouvernement; étude fatigante, et dont on n'est capable que dans la première jeunesse, et peut être dans les pays où l'estime publique nous promet beaucoup d'avantages ${ }^{30}$. L'expression séduit néanmoins. Et déjà par l'autonomie du pouvoir de la raison qu'elle paraît consacrer. En Angleterre, cette préoccupation est apparue au milieu du XIX ${ }^{\text {ème }}$ siècle comme en témoignent les controverses entre James Mill (Essays on Government en 1828), Mac Caulay et John Stuart Mill (A System of Logic, en 1843) ${ }^{31}$. Et, plus encore, l'action des cercles benthamites emmenés par Charles Trevelyan (qui sera sous-secrétaire d'État à la Trésorerie et auteur, avec Stafford Northcote, en 1853 du rapport sur la réforme du Civil Service demandé par Gladstone au nom d'une classe moyenne soucieuse de développer un recrutement au mérite) ${ }^{32}$. Du coup, l'objet «administration publique » a longtemps

28. Osborne (D.) et Gaebler (T.), Reinventing Government: How the Entrepreneurial Spirit is Transforming the Public Sector, Reading, Mass.; Don Mills, Ont., Addison-Wesley Pub., 1992.

29. Sur ces figures de savants et d'artisans, voir Taylor (Eva G.R.), The Mathematical Practitioners of Tudor and Stuart England, Cambridge, Cambridge University Press, 1954.

30. De l'esprit, Paris, Durand, 1758, p. 643.

31. Sur ce point, voir Kort (F.), «The Issue of a Science of Politics in Utilitarian Thought ", The American Political Science Review, 46, 4, décembre 1952, p. 1140-1152.

32. Hughes (E.), "Civil Service Reform, 1853-1855», Public Administration, cité par Françoise Dreyfus, qui analyse la mise en cuvre de cette réforme dans L'invention de la bureaucratie. Servir l'État en France, en Grande-Bretagne et aux États-Unis (XVII ème -XIX ${ }^{\text {ème }}$ siècles), Paris, La Découverte, 2000, p. 170 et $s$. 
semblé insuffisant pour, dans ce pays, donner lieu à une formulation spécifique au sein des « sciences du politique ». Le culte de l'amateurisme, l'absence de centralisation, la faiblesse relative de l'ethos bureaucratique y ont contrarié l'essor de telles spécialisations. Sur le continent, en revanche, les configurations académiques étaient différentes. Révéler des régularités dans le flux apparemment incohérent des faits sociaux, créer les bases d'une cumulativité des expériences administratives, comprendre et conquérir l'« opinion » : le réaménagement des savoirs y disposait des incitations pour se « durcir » progressivement. Il s'agissait de « réinventer» le gouvernement par une critique de son organisation et un programme de régénération interne. D'autant que l'existence d'académies spécialisées et de pensions d'État venaient structurer un «marché » des revendications de scientificité déjà relativement autonome ${ }^{33}$. L'abbé Grégoire est sans doute celui qui a manifesté avec le plus d'optimisme cette revendication. Il en présente le tableau enthousiaste, à l'Institut, devant ses collègues de la Classe des sciences morales et politiques en 1796. Si le gouvernement représentatif peut être défini comme « commis » à la défense des «droits liés de la liberté et du bonheur », c'est parce qu'il s'appuie sur l'étude de la « science de gouvernement ${ }^{34}$. Et cela bien que cette première académie française spécialisée dans la recherche en sciences sociales ait surtout perfectionné entre 1795 et 1803 les moyens d'une surveillance politique ${ }^{35}$.

Dans une Europe que bouleversent le principe des nationalités, le mouvement d'industrialisation ou l'arrivée des masses sur la scène électorale, la revendication est une manière - il ne faut pas s'y tromper - de faire de nécessité vertu. Accroître la puissance d'un État, c'est multiplier le nombre de ses sujets, c'est assurer leurs richesses et leur bonheur, c'est éviter les guerres étrangères, justifier celles qui sont indispensables. Mais pour mener de telles tâches, encore faut-il maîtriser les arcanes des savoirs qui justifient le principe d'une compétence à gouverner, et donc d'un droit à dominer. La «science du gouvernement » devient dès lors la condition d'évaluation de ce qui doit être poursuivi comme le «bien de l'État» ou la «prospérité de la communauté politique ». L'essor de l'économie politique va lui donner ses lettres de noblesse. Avant qu'elle ne se déploie à partir de la fin du XIX ${ }^{\text {ème }}$ siècle dans d'innombrables directions, au gré de la redéfinition des missions mêmes de l'État : l'administration des colonies, la prévention des risques naturels, la lutte contre l'insalubrité, la codification de la « régularité » et «sincérité » des scrutins, la formation des élus locaux, la «police» des familles, la récompense des actes d'héroïsme ou la propagande internationale... Des principes d'intervention mais aussi des techniques d'objectivation forgés par des groupes de savants comme par des réseaux professionnels et militants et qui, circulant de pays en pays, prétendaient maximiser l'efficacité du travail bureaucratique ${ }^{36}$. Peut-on soutenir

33. Sur ces « marchés scientifiques ", voir Gillespie (C.- C.), Science and Polity in France at the End of The Old Regime, Princeton (N. J.), Princeton University Press, 1981.

34. Réflexions... sur les moyens de perfectionner les sciences politiques. Mémoires de l'Institut national des sciences et des arts. Classe des sciences morales et politiques, T. I, Paris, an VI, p. 552 et s.

35. Sur l'histoire sociale de cette Académie qui ne peut être rappelée ici, voir Staum (M.), « The Class of Moral and Political Sciences, 1795-1803», French Historical Studies, 11, 1980, p. 371-97.

36. Par objectivation, il faut entendre les « processus qui contribuent à inscrire les relations sociales et les règles en usage dans ces relations dans l'ordre d'une «réalité objective » c'est-à-dire perçue comme extérieure aux individus s'imposant à eux et régissant leurs comportements », Lagroye (J.), Sociologie politique, Paris, Presses de la FNSP et Dalloz, 1997, ( $3^{\text {èm }}$ éd.), p. 157. Sur l'utilisation de cette notion dans l'analyse des processus politiques, voir Lacroix (B.), «Ordre politique et ordre social. Objectivisme, objectivation et analyse politique », in : Grawitz (M.), Leca (J.) (dir.), Traité de science politique, Paris, PUF, T. 1,1985 , p. $469-565$. 
toutefois que tout est expérimental et - utilisons le mot - positif dans ces sciences de gouvernement ? Assurément non. Mais c'est précisément ce qui donne de l'intérêt à la découverte de ces savoirs pratiques. Jetées dans le siècle, avec la promesse de « rendre l'esprit plus clairvoyant, sinon de dissiper les préjugés », les semences de ces « sciences utiles ${ }^{37}$ permettent de rompre avec la conception mythologique et souvent anachronique de l'histoire de la science politique.

\section{FORMES ET USAGES DE L'ACADÉMISME}

Que la connaissance savante de l'exercice du pouvoir ait une histoire, nul n'en doute. Mais celle-ci a trop souvent les allures d'une mémoire, à la fois linéaire et enchantée. Quentin Skinner pointerait sans doute un fautif: la «mythologie de la cohérence ", ce principe de narration cher aux historiens des idées qui consiste à transformer des remarques éparses ou incidentes en "doctrine » constituée. Signe que sur la base d'une similitude de terminologie ou d'une filiation supposée, l'exégèse des textes du passé sait développer son propre cérémonial ${ }^{38}$. Mais la mise en garde mérite d'être élargie. Reconstruite par chaque nouvelle génération (au gré des rapports de force entre producteurs accrédités de ces formes de savoir ${ }^{39}$ ), l'écriture disciplinaire risque constamment de rencontrer deux autres écueils. Et bien plus importants. Celui du présentisme - rendre compte du passé à partir du seul point de vue qu'offre la situation du moment présent - ; celui aussi, quoique moins visible, de l'académisme — rendre compte du passé dans les termes des partages disciplinaires et des principes de reconnaissance autour desquels se construit de nos jours sa légitimité institutionnelle.

Pour s'affranchir de ces évidences rétrospectives, l'étude de la disciplinarisation du savoir doit faire droit aux processus, aux tâtonnements, aux équivoques qui l'ont accompagnée. Elle doit revenir sur l'examen empirique des conditions d'institutionnalisation des « sciences » qui ont pris le gouvernement des corps et des esprits pour objet. En traquer les conditions d'expansion, c'est interroger le lien entre la bureaucratisation des savoirs et le développement de la grande industrie ou la redéfinition des rapports entre âges, sexes et groupes sociaux. C'est éclairer ses liens avec l'affirmation de l'État, la nationalisation et la parlementarisation du politique. L'autonomie méthodologique de ces savoirs n'est pas donnée a priori : elle se construit dans et par les concurrences qui n'ont pas cessé de nourrir depuis le début du XIX ${ }^{\text {ème }}$ siècle la rationalisation de

37. L'expression est empruntée à Cesare Lombrose, Le crime politique et les révolutions, par rapport au droit, à l'anthropologie criminelle et à la science du gouvernement, trad. de A Bouchard, Paris, Félix Alcan, 1892 , T. 1, p. 30. Plus généralement, sur l'essor social de la criminologie « républicaine ", voir l'ouvrage de Kaluszynski (M.), La République à l'épreuve du crime. La construction du crime comme objet politique 1880-1920, Paris, LGDJ, 2002. p. $3-53$.

38. Skinner (Q.), « Meaning and Understanding in the History of Ideas », History and Theory, 8 (1969),

39. Sur les principes de construction de la discipline «science politique » en France, c'est-à-dire des « processus et dispositifs qui tendent à discipliner ceux qui participent à l'élaboration de ce savoir particulier », tel qu'il s'appréhende notamment au travers de ses congrès académiques, avec ses « positions de savoir » et ses «positions de pouvoir», voir Lagroye (Jacques), «La science politique comme discipline », Actes de la recherche en sciences sociales, à paraître. Nous tenons à remercier Jacques Lagroye de nous avoir permis de bénéficier des résultats de sa recherche, d'autant que ces quelques pages lui doivent beaucoup. 
l'exercice du pouvoir. D'où une double interrogation. Comment la production de savoirs en matière politique débouche-t-elle sur des « sciences d'administration », voire sur des «sciences d'État» 40 ? Comment furent-elles légitimées puis mises en œuvre par des administrations publiques confrontées à des problèmes spécifiques ?

Le point vaut qu'on y insiste tant l'illusion est tenace : l'autonomie des productions savantes qui ont pris l'action gouvernementale pour objet est davantage proclamée que démontrée. Ce qui anime ces producteurs, c'est moins de savoir que d'agir. C'est dire si leurs efforts ne visent pas qu'à répondre à des exigences désintéressées. Chaque découpage disciplinaire est fruit de rivalités autant sociales qu'intellectuelles ${ }^{41}$. Charles Coulston Gillispie l'avait souligné à propos de la Société royale de Londres ou de l'Académie des Sciences à Paris : le passage au XVIII ${ }^{\text {ème }}$ siècle des comités d'amateurs à des structures centralisées et professionnalisées doit beaucoup à un éthos scientifique que l'émulation d'État a faconné ${ }^{42}$. Par tout un jeu de signes de reconnaissance et de gratifications hiérarchiques. L'exhibition de connaissances (sinon leur définition) reste ainsi inséparable des usages qui en sont attendus. Et déjà des titres d'excellence qui leur sont liés. Que l'on songe à La guerre des médailles de Jean Delsarte au CNRS qui, en 1937, fut le principal artisan de la fronde contre le projet de système de récompenses pécuniaires et honorifiques conçu par le physicien Jean Perrin, créateur du CNRS et alors premier sous-secrétaire d'État à la recherche scientifique. De nos jours, c'est au nom d'une «culture de l'évaluation» que le projet s'étend. Avec l'idée de «motiver» le monde de la recherche par des « récompenses » venant rémunérer le «mérite » de ceux qui déposent des brevets, transfèrent des technologies ou multiplient leur collaboration avec les milieux administratifs et industriels ${ }^{43}$. Entre science et gouvernement, une forme caractéristique de relation s'est établie. Elle consiste moins, pour reprendre le vocabulaire de Gillespie, en un partisanship qu'en un partnership. Pour les scientifiques, les pouvoirs publics sont synonymes de ressources et de reconnaissance institutionnelle. Pour les gouvernants, les savants sont sources d'expertise et de légitimation. Ce qui est attendu sur le terrain spécifique de l'action publique ? Tout à la fois de "découper » les problèmes en domaines sectoriels spécialisés, d'élaborer des procédures d'évaluation et d'intervention plus fiables, de justifier des revirements de ligne de conduite, de stabiliser fonctionnellement des territoires d'action, d' «interpréter » les demandes adressées aux autorités publiques, privées et associatives, au gré des circonstances et des formes de coopération engagées. Ce sont précisément ces savoir-faire mais surtout leur compréhension pratique que les acteurs professionnels, tant publics que privés, ont appris à maîtriser. D'où leur « prix » sur le terrain de la conduite des dispositifs d'action publique

40. Bourdieu (P.), Christin (O.), Will (P.-E.), « Sur la science de l'État », Actes de la recherche en sciences sociales, 133 , juin 2000 , p. 3-9.

41. Pour une démonstration qui souligne la tendance des sciences sociales étatsuniennes à délaisser l'examen des "causes des phćnomènes sociaux " (causation of social phenomena) au profit des "effets des interventions sociales », voir Richard P. Nathan, Social Science in Government: Uses and Misuses, New York, Basic Books, 1988. L'auteur, politiste de la Woodrow Wilson School à l'Université de Princeton, fut de 1972 à 1979 membre du bureau de la célèbre Brooking Institution et partic prenante des «états-majors 》du gouvernement fédéral.

42. Science and Polity in France at the End of the Old Regime, Princeton (N. J.), Princeton University Press, 1981, notamment p. 80 et s.

43. Les Échos du 11 décembre 2002. Sur cette instrumentation pécuniaire et honorifique des savoirs à vocation scientifique, voir Barbara F. Reskin, «Academic Sponsorship and Scientist's Careers », Sociology of Education, vol. 52, 3, juillet 1979, p. 129-146 et pour une application à un secteur spécialisé, celui des physiciens, Charles Derber, "Sponsorship and the Control of Physicians ", Theory and Society, vol. 12, 5 , septembre 1983, p. 561-601. 
et des instruments de «pilotage» qui leur sont associés au niveau local, national ou diplomatique.

Ce qui rend possible le projet d'une science de gouvernement, c'est donc avant tout l'idée (pourtant bien problématique) d'une continuité épistémologique des questions relatives au fonctionnement du gouvernement avec les interrogations des sciences en général. Une telle croyance nourrit depuis longtemps une vision pyramidale des productions savantes, étalonnée par leur degré d'utilité sociale. Pour le publiciste Auguste Billard, « la science de l'administration, que l'on croit si facile, si bornée, est au contraire la plus étendue de toutes les sciences, celle qui demande le plus d'études; elle n'a de réalité que par l'application qu'on en peut faire aux besoins de la socićté » ${ }^{44}$. Et de regretter que l'Académie des sciences morales et politiques se tienne, en matière de science sociale, non point en tête, mais «à côté si ce n'est en arrière du mouvement général des esprits». Seconde condition : disposer du crédit nécessaire pour former certains publics à telle ou telle ingénierie du politique, que ce soit à l'échelle locale ou au plan national, voire dans des organismes internationaux. Le projet n'a pas perdu de son actualité : «mieux préparer» les collaborateurs d'élus locaux et nationaux, les administrateurs mais aussi les cadres d'entreprises publiques et privées reste un souci de nombre de «spécialités » académiques. Surtout depuis que les «politiques publiques » et plus généralement les formes d'action administrative ont connu ces dernières décennies d'importants bouleversements. Qu'il s'agisse des transformations économiques et sociales qui, depuis la crise du milieu des années 1970, ont généré la "mondialisation », ou des formes d'intégration régionale qui ont mis à mal les schémas classiques du «pilotage » de l'action publique. Une double remise en cause en a résulté : au niveau des territoires sur lesquels s'appliquent ces « régulations » de plus en plus complexes et dissociées; au niveau des modes d'intervention et des catégories d'action utilisés par les pouvoirs exécutifs (rôle accru de l'opinion publique, formes renouvelées de partenariat et de concertation, décentralisation des niveaux de compétence, redéfinition des modèles de légitimation, etc.). D'où les perspectives ouvertes par ce chantier de recherche. En s'attachant à l'objectivation des « sciences de gouvernement », il fait lever un espoir: celui de mieux comprendre les formes que les savoirs académiques sur l'action publique peuvent prendre, les usages sociaux dont ils ont été l'objet, les acteurs et matériaux qui les ont portés, les échecs et réussites qu'ils ont connus.

\begin{abstract}
Annexe
Plusieurs thèses de science politique soutenues récemment ou en cours de réalisation au sein du CERAT (CNRS) à l'IEP de Grenoble ont balisé les attendus liés à cette thématique de recherche, soit dans le cadre d'une critique des fondements de la citoyenneté républicaine et de ses modes de représentation, soit au titre d'une socio-histoire des formes de l'action publique. À l'heure où les anathèmes contre le travail en laboratoire se font à nouveau entendre, il faut redire la force de la cumulativité scientifique et de l'échange structuré par des rapports d'équipe, même si bien entendu chaque recherche garde sa propre cohérence problématique et se positionne sur un «front » distinct de la science sociale (histoire des sciences, sociologie des politiques publiques, histoire sociale des professions, anthropologie
\end{abstract}

44. Art. «Académie » du Dictionnaire de la politique. Encyclopédie du langage et de la science politiques, publié par E. Duclerc et Pagnerre, avec une introduction de Garnier Pagès, Paris, Pagnerre, 1860, p. 27. Voir aussi son article « Administrateur» dans le même volume (p. 48). 
de l'État, philosophie juridique). C'est pourquoi, à défaut d'une présentation détaillée, on rappellera ici tout l'intérêt des travaux de :

- Isabelle Benoît, Politiques de mémoire. Les musées d'histoire en France et en Allemagne 1945-1995, Thèse de science politique de l'Institut européen de Florence et de l'IEP de Grenoble, 2000.

- Rachel Vanneuville, La référence anglaise à l'École libre des sciences politiques. La formation de gentlemen républicains, 1871-1914, Thèse de science politique, I.E.P. de Grenoble, 1999.

- Véronique Dimier, Construction et enjeux d'un discours scientifique sur l'administration coloniale en France et en Grande-Bretagne (1930-1950), Thèse de science politique, I.E.P de Grenoble, 1999.

- Vincent Spenlehauer, L'évaluation des politiques publiques, avatar de la planification, Thèse de science politique, I.E.P. de Grenoble, 1998.

- Damien Deschamps, La République aux colonies: le citoyen, l'indigène et le fonctionnaire. Citoyenneté, cens civique et représentation des personnes, le cas des Établissements français de l'Inde et la genèse de la politique d'association (vers 1848, vers 1900), Thèse de science politique, I.E.P. de Grenoble, 1998.

- Frédéric Caille, Les instruments de la vertu. L'État, le citoyen et la figure du sauveteur en France: construction sociale et usages politiques de l'exemplarité morale, de la fin de l'Ancien Régime à 1914, Thèse de science politique, I.E.P. de Grenoble, 1997.

- Anne Verjus, Les femmes épouses et mères de citoyens ou de la famille comme catégorie politique dans la construction de la citoyenneté (1789-1848), Thèse de doctorat commencée au CERAT à l'IEP de Grenoble mais soutenue à l'EHESS (1997).

- Philippe Veitl, Les régions économiques. Clémentel et l'invention de la région des Alpes françaises, Thèse de science politique, I.E.P. de Grenoble, 1992.

Il faudrait aussi signaler plusieurs recherches en cours :

- Anne-Laure Guyot, Les modes de régulation des relations entre la justice judiciaire et le politique. Analyse politique comparée des cas allemand, français, italien.

- Renaud Payre : À la recherche d'une "science communale». Les "mondes" de la réforme municipale dans la France de la première moitié $d u X X^{\text {ème }}$ siècle (thèse CIDSP, IEP Grenoble).

- Guillaume Marrel, Sociologie et histoire du cumul des mandats en France. Le cas des députés maires urbains (1848-1958).

- Nathalie Dompnier, La clef des urnes. La construction sociohistorique de la déviance électorale en France depuis 1848 (thèse CIDSP, IEP Grenoble). 